\title{
Three-dimensional gravity modelling and focusing inversion using rectangular meshes
}

\author{
Michael Commer \\ Lawrence Berkeley National Laboratories \\ Earth Sciences Division \\ 1 Cyclotron Road, MS 90-1116 \\ Berkeley, CA 94720 \\ $U S A$
}

(March 17, 2011)

\begin{abstract}
Rectangular grid cells are commonly used for the geophysical modeling of gravity anomalies, owing to their flexibility in constructing complex models. The straightforward handling of cubic cells in gravity inversion algorithms allows for a flexible imposition of model regularization constraints, which are generally essential in the inversion of static potential field data. The first part of this paper provides a review of commonly used expressions for calculating the gravity of a right polygonal prism, both for gravity and gradiometry, where the formulas of Plouff and Forsberg are adapted. The formulas can be cast into general forms practical for implementation. In the second part, a weighting scheme for resolution enhancement at depth is presented. Modeling the earth using highly digitized meshes, depth weighting schemes are typically applied to the model objective functional, subject to minimizing the data misfit. The scheme proposed here involves a non-linear conjugate gradient (NLCG)
\end{abstract}


inversion scheme with a weighting function applied to the NLCG scheme's gradient vector of the objective functional. The low depth resolution due to the quick decay of the gravity kernel functions is counteracted by suppressing the search directions in the parameter space which would lead to near-surface concentrations of gravity anomalies. Further, a density parameter transformation function enabling the imposition of lower and upper bounding constraints is employed. Using synthetic data from models of varying complexity and a field data set, it is demonstrated that, given an adequate depth weighting function, the gravity inversion in the transform space can recover geologically meaningful models requiring a minimum of prior information and user interaction. 


\section{Introduction}

Gravity instruments measure the vertical component of the Earth's gravitational attraction. Gravity gradiometry measures the gradient of a gravity field. Simulations of both types of measurements over complex model geometries can be accomplished with relatively little effort using highly digitized rectangular grids. Three-dimensional (3D) regularized pixel-based inversions where right rectangular prisms define unknown density parameters have been a popular approach (Li and Oldenburg, 1998; Boulanger and Chouteau, 2001; Zhdanov et al., 2004; Dias et al., 2009). Much effort has gone into inversion algorithms that counteract the effects due to the poor depth resolution of gravity data while producing sharp images which at the same time exhibit the true source depths of gravity anomalies. Early approaches involve modeling gravity anomalies by means of bodies with uniform density, where geometric shape parameters are to be optimized either interactively or automatically (Zidarov and Zhelev, 1970; Oldenburg, 1974; Pedersen, 1977); along these lines are also compactness (minimum volume) criteria (Last and Kubik, 1983). With sufficient background information, one can initiate the inversion process such that anomaly depths and structures are systematically expanded consistent with known geology (Rene, 1986; Camacho et al., 2000; Bosch et al., 2006). With limited prior knowledge, focusing stabilizers are beneficial (Zhdanov et al., 2004). Alternatively, an interpreter-guided inversion (Silva and Barbosa, 2006) can still produce sharp images by initializing multiple inversions with compact anomaly sources in a trial-and-error way or by using adaptive-learning techniques (Dias et al., 2009).

The combination of Tikhonov regularization methods with procedures that penalize near-surface mass concentrations, have the large advantage of requiring a minimum of prior 
knowledge (Li and Oldenburg, 1998). The approach described here aims at producing physically realistic density models with a minimum amount of prior information and a minimum set of user-defined control parameters. The obvious drawback of producing rather diffuse images is accepted in exchange for the method's simplicity. Two types of applications are considered. First, to deliver starting models for interpreter-guided gravity inversions, where sharp boundaries may be introduced. Second, gravity data are often included into joint inversion frameworks with different geophysical attributes. A popular method for the joint inversion of gravity data with data types that are sensitive to different geophysical attributes, for example magnetic properties, involves equality constraints for structural similarity resemblance (Fregoso and Gallardo, 2006). Seeking to identify structural resemblance between different attributes, subject to fitting all included data types, it may not be desirable to impose structural constraints which are justified by only the gravity data.

For completeness, the first part of this paper presents the various formulas for calculating gravity and gradiometry right rectangular prism contributions in a practical way that facilitates implementation. A class of direct methods exists for calculating gravity anomalies of more complex uniform polyhedral bodies, which have been analyzed thoroughly and improved to alleviate limitations due to numerical rounding errors (Holstein and Ketteridge, 1996). Most 3D gravity inversions, including the one presented here, employ prismatic elements. Li and Chouteau (1998) present a comprehensive history of the different derivations of analytical formulas for right rectangular prisms, with an emphasis on their validity and singularity issues. I chose to focus on the formulas of Plouff (1976) and Forsberg (1984), since they cause less difficulties with singularities for interior points. After first revisiting the approximate formulations typically used in 3D pixel-based inversions, corresponding exact analytical formulas are summarized. It is also shown in the appendix that Forsberg's 
expressions for the off-diagonal gradiometry tensor elements may be recast into a more compact formula.

The second part of this paper introduces the two main components of the presented gravity inversion method. The first is a method for enhancing model resolution by reweighting the gradient of the data misfit objective functional in a gradient-based inversion method. Stabilized gradient-based inversion methods have proved to be efficient for 3D imaging problems with highly parametrized models. The principal difference of the proposed gradient weighting scheme to other approaches (Li and Oldenburg, 1998; Zhdanov, 2002 ) is that it re-weights, in a mathematically less rigorous way, the data misfit objective functional gradient vector, rather than re-weighting the stabilizing counterpart. Second, a parameter transformation function originally employed in inversions for electrical conductivity (Commer and Newman, 2008), is adapted for the straightforward imposition of lower and upper density bounds. In the application part of this paper, the effectiveness of the gradient weighting method in counteracting the tendency of concentrating anomalies near observation points is demonstrated on synthetic data from simple models and a more complex large-scale model from the Gulf of Mexico. Also, inverting in the transformed space is analyzed on synthetic models. Finally, the method's usefulness is tested on a field data set from the mining industry.

\section{Right rectangular prism solutions}

Consider a rectangular cell with edge coordinates $\left(x_{1}, x_{2}\right),\left(y_{1}, y_{2}\right)$, and $\left(z_{1}, z_{2}\right)$ along the $x, y$, and $z$ axes of a right-handed Cartesian system, where the $z$-axis points downward. Its vertical gravitational attraction contribution at an observation point $\mathbf{r}=(x, y, z)$ is 
represented by the integral (Zhdanov, 1988)

$$
\mathbf{g}(\mathbf{r})=\gamma \int_{z_{1}}^{z_{2}} \int_{y_{1}}^{y_{2}} \int_{x_{1}}^{x_{2}} \varrho\left(\mathbf{r}^{\prime}\right) \frac{\mathbf{r}^{\prime}-\mathbf{r}}{\left|\mathbf{r}^{\prime}-\mathbf{r}\right|^{3}} d x^{\prime} d y^{\prime} d z^{\prime}
$$

In (1), $\gamma$ is Newton's gravitational constant and $\varrho\left(\mathbf{r}^{\prime}\right)$ is the density distribution at the position $\mathbf{r}^{\prime}=\left(x^{\prime}, y^{\prime}, z^{\prime}\right)$. The density is assumed to be constant over the cell volume. Considering the vertical gravity contribution $g_{z}$ of an infinitesimal volume element, $d V^{\prime},(1)$ becomes

$$
g_{z}=\gamma \varrho \frac{z^{\prime}-z}{\left|\mathbf{r}^{\prime}-\mathbf{r}\right|^{3}} d V^{\prime}
$$

By substituting $a=x^{\prime}-x, b=y^{\prime}-y$, and $c=z^{\prime}-z$, the following triples are introduced:

$$
\begin{aligned}
& \ell=1:(a, b, c)_{1}=\left(y^{\prime}-y, z^{\prime}-z, x^{\prime}-x\right), \\
& \ell=2:(a, b, c)_{2}=\left(z^{\prime}-z, x^{\prime}-x, y^{\prime}-y\right), \\
& \ell=3:(a, b, c)_{3}=\left(x^{\prime}-x, y^{\prime}-y, z^{\prime}-z\right),
\end{aligned}
$$

and will be reused further below. Denoting $g_{z}$ as $g_{\ell}$ with $\ell=3$, a more general notation for (2) is

$$
g_{\ell}=\gamma \varrho \frac{c}{\left|\mathbf{r}^{\prime}-\mathbf{r}\right|^{3}} d V^{\prime}
$$

In other words, $g_{x}=g_{1}(\ell=1)$ is equivalent to the vertical gravity, when the $x$ coordinate becomes the vertical coordinate, and the other two coordinates in (3) shift accordingly to the left. Similarly, one obtains $g_{y}=g_{2}(\ell=2)$ when shifting the triple entries twice to the left. Obviously, a shift of 3 for $\ell=3$ has no effect. This scheme is proposed here for an easy numerical implementation using array index shifting, and might be more useful for the gradiometry tensor elements (Zhdanov et al., 2004)

$$
\begin{aligned}
g_{\ell \ell} & =\gamma \varrho\left[\frac{3 c^{2}}{\left|\mathbf{r}^{\prime}-\mathbf{r}\right|^{5}}-\frac{1}{\left|\mathbf{r}^{\prime}-\mathbf{r}\right|^{3}}\right] d V^{\prime}, \\
g_{\ell \ell^{\prime}} & =\gamma \varrho \frac{3 a b}{\left|\mathbf{r}^{\prime}-\mathbf{r}\right|^{5}} d V^{\prime}, \quad \ell \neq \ell^{\prime},
\end{aligned}
$$


where, as before, $\ell, \ell^{\prime}=1,2,3$ refers to the $x, y$, and $z$ coordinates, respectively.

The closed-form for the integral (1) has been derived by several authors (Nagy, 1966; Okabe, 1979; Plouff, 1976) in order to compute the vertical gravity component $g_{z}$. Holstein et al. (1999) provide a comprehensive comparison between representative closed formulas applicable to polyhedral density anomalies. For this work, the representation given by Plouff is adapted, taking advantage of its straightforward implementation,

$$
\begin{aligned}
g_{z} & =\gamma \varrho \int_{z_{1}}^{z_{2}} \int_{y_{1}}^{y_{2}} \int_{x_{1}}^{x_{2}} \frac{z^{\prime} d x^{\prime} d y^{\prime} d z^{\prime}}{r^{3}} \\
& =\gamma \varrho \sum_{i=1}^{2} s_{i} \sum_{j=1}^{2} s_{j} \sum_{k=1}^{2} s_{k}\left[z_{k} \arctan \frac{x_{i} y_{j}}{z_{k} r_{i j k}}-x_{i} \ln \left(r_{i j k}+y_{j}\right)-y_{j} \ln \left(r_{i j k}+x_{i}\right)\right]
\end{aligned}
$$

with $s_{1}=-1, s_{2}=+1$. The indices $i, j$, and $k$ refer to the cell corners along the $x, y$, and $z$ coordinates. For simplicity, the observation point is at the origin in this formulation. Then, one has $r_{i j k}=\sqrt{x_{i}^{2}+y_{j}^{2}+z_{k}^{2}}$, the distance from the observation point to a corner $(i, j, k)$. The similar formula of Okabe (1979) also involves an arc-tangent expression in the first term within brackets in (9). The equivalent of (9) derived earlier by Nagy (1966) leads to an arc-sine expression, where in his paper the terms resulting from the integration over the cube volume are explicitly written out*.

Plouff's formulation, now written with respect to an observation point $\mathbf{r}=(x, y, z)$, can be generalized similarly to (6) in order to facilitate an implementation including all three gravity components $(\ell=1,2,3)$ :

$$
\begin{aligned}
g_{\ell} & =\gamma \varrho \int_{a_{1}}^{a_{2}} \int_{b_{1}}^{b_{2}} \int_{c_{1}}^{c_{2}} \frac{c d z d y d x}{r^{3}} \\
& =\gamma \varrho \sum_{i=1}^{2} s_{i} \sum_{j=1}^{2} s_{j} \sum_{k=1}^{2} s_{k}\left[c_{k} \arctan \frac{a_{i} b_{j}}{c_{k} r_{i j k}}-a_{i} \ln \left(r_{i j k}+b_{j}\right)-b_{j} \ln \left(r_{i j k}+a_{i}\right)\right],
\end{aligned}
$$

*When adapting Nagy's integration written out over 16 lines in his equation (8), note that the equation's first arc-sine argument (3rd equation line) should read $y_{2} r$ instead of $y_{2}^{2} r$ in the enumerator. Further, the last square root expression in line 13 of the same equation should read $\sqrt{x_{1}^{2}+y_{1}^{2}+z_{2}^{2}}$ instead of $\sqrt{x_{1}^{2}+y_{2}^{2}+z_{1}^{2}}$. 
where now $r_{i j k}=\sqrt{a_{i}^{2}+b_{j}^{2}+c_{k}^{2}}$. In equation (10), the coefficients $a, b$, and $c$ are distances from the prism corners to the observation point $\mathbf{r}$. Again, putting these coefficients into a triple, $\left(a_{n}, b_{n}, c_{n}\right)_{\ell}$ with $n=1,2$, the three values $g_{\ell}$ can be easily computed by a cyclic shift,

$$
\begin{aligned}
& \ell=1:\left(a_{n}, b_{n}, c_{n}\right)_{1}=\left(y-y_{n}, z-z_{n}, x-x_{n}\right), \\
& \ell=2:\left(a_{n}, b_{n}, c_{n}\right)_{2}=\left(z-z_{n}, x-x_{n}, y-y_{n}\right), \\
& \ell=3:\left(a_{n}, b_{n}, c_{n}\right)_{3}=\left(x-x_{n}, y-y_{n}, z-z_{n}\right) .
\end{aligned}
$$

The same scheme can be applied to the diagonal gradiometry tensor components, which were introduced by Forsberg (1984), see also Li and Chouteau (1998). With $g_{x x}=g_{11}$, $g_{y y}=g_{22}$, and $g_{z z}=g_{33}$, and the shifting rules (11)-(13), one obtains

$$
g_{\ell \ell}=\gamma \varrho \sum_{i=1}^{2} s_{i} \sum_{j=1}^{2} s_{j} \sum_{k=1}^{2} s_{k} \arctan \frac{a_{i} b_{j}}{c_{k} r_{i j k}}
$$

Similarly, the off-diagonal gradiometry tensor elements can be written as $\left(\ell, \ell^{\prime}=1,2,3\right.$ and $\left.\ell \neq \ell^{\prime}\right)$

$$
g_{\ell \ell^{\prime}}=-\gamma \varrho \sum_{i=1}^{2} s_{i} \sum_{j=1}^{2} s_{j} \sum_{k=1}^{2} s_{k} \ln \left(c_{k}+r_{i j k}\right),
$$

where $g_{y z}=g_{23}$ uses rule (11), $g_{x z}=g_{13}$ uses rule (12), and $g_{x y}=g_{12}$ uses rule (13), and further $g_{\ell \ell^{\prime}}=g_{\ell^{\prime} \ell}$. For the discussion of a singularity issue arising for $g_{\ell \ell^{\prime}}$, and a more compact representation, the reader is referred to the appendix.

Figure 1 illustrates the gravity effect for $g_{z}$ (a) and the gradiometry effect for $g_{z z}$ (b) and $g_{x y}(\mathrm{c})$ using the standard cubic model of Li and Chouteau (1998). The cube has the unit density of $1 \frac{\mathrm{g}}{\mathrm{cm}^{3}}$ over a volume defined by the corner positions $\pm 10 \mathrm{~m}$ for each Cartesian coordinate. The figure also shows where the effects are zero, indicated by the dotted planes in (a) and (c), and the diagonal dotted lines in (b), connecting opposite 
corners of the shown volume. A FORTRAN90 double precision implementation was used for these calculations. The extremal gravities occur at the center of each cube face and are $g_{\ell=1,2,3}=+/-346.561 \mu \mathrm{Gal}$ for $x, y, z=-/+10 \mathrm{~m}$. The same value can be obtained from formula (6), where the standard cube model was recursively split into smaller sub cubes until the absolute difference between (6) and (10) dropped below $2 \cdot 10^{-10} \mu$ Gal. Using their implementation of (10), Li and Chouteau (1998) report an extremum of $346.426 \mu \mathrm{Gal}$ for these positions. Exemplified for $g_{z z}$ in Figure 1b, the positive extrema occur close to the corner points, while the negative extrema occur when approaching the centers of the horizontal prism edges. Further, the extremal values of $g_{x y}$ are encountered when approaching the prism corners (c).

Employing (6) without splitting cells located in the vicinity of the observation point can cause a high approximation error. The numerical error is quantified in Figure 1 (d-f). All calculated positions with a difference of at least $10 \%$ between the values obtained from the approximations (6), (7), and (8) and their respective closed form solutions (10), (14), and (15) are shown, where only absolute values of $g_{\ell}, g_{\ell \ell}$, and $g_{\ell \ell^{\prime}}$ with a magnitude of at least $10 \%$ of the extreme are considered. The figure shows that the numerical error only becomes an issue within approximately a cell diameter. However, this has to be taken into account when for example modeling near-surface anomalies or borehole gravity.

\section{3-D gravity least-squares inversion}

The gravity inverse problem is formulated by the minimization of the Tikhonov parametric functional to be minimized

$$
\Phi=\Phi_{d}+\lambda \Phi_{m}=\frac{1}{2}\left[\mathbf{D}\left(\mathbf{d}^{o}-\mathbf{d}^{p}\right)\right]^{2}+\frac{1}{2} \lambda(\mathbf{W} \mathbf{m})^{2} \rightarrow \min
$$


This functional for real-valued data vectors, d, where the superscripts $o$ and $p$ refer to observed and calculated (or predicted) data, respectively, has been adapted from a nonlinear conjugate gradient (NLCG) optimization scheme for complex electromagnetic data (Newman and Alumbaugh, 1997; Commer and Newman, 2008). The calculated data are obtained through a forward modeling operator $F(\mathbf{m})=\mathbf{d}^{p}$, where the model vector $\mathbf{m}$ represents either absolute densities or density anomalies with respect to a background density. To calculate $\mathbf{d}^{p}$, the formulations (10), (14), and (15) were used for all shown results. The relatively high computing effort caused by the arc-tan and logarithmic functions in these formulations, aggravated when using highly digitized finite-difference meshes, is addressed by a parallel implementation of the forward modeling algorithm. Using the Message Passing Interface, a Cartesian communicator topology is used such that each parallel task computes a sub-cube of the model domain. The parallel forward modeling algorithm only requires message passing when summing up the gravity contributions from each sub cube. Similarly, the only inter-process communication required by the NLCG method is given by a few global dot products for updating the NLCG search directions.

During the iterative inversion process, the diagonal weighting matrix $\mathbf{D}$ is applied to the differences between observed and calculated data in order to eliminate the influence of data below noise level. Following Li and Oldenburg (1998), uncorrelated Gaussian noise of $0.05 \mathrm{mGal}$ and 2 percent of the datum's absolute value has been added to the synthetic inversion studies of this work.

The second term of (16) contains the regularization parameter $\lambda$, for weighting the stabilizing term, $\mathbf{W m}$, where $\mathbf{W}$ approximates a Laplacian operator on the finite-difference grid (Newman and Alumbaugh, 1997). For inverse problems with uncertain noise estimates, one can determine a proper value of $\lambda$ using generalized cross-validation and L-curve criteria 
(Li and Oldenburg, 2003; Farquharson and Oldenburg, 2004).

\section{Gradient depth weighting for focusing inversion}

To counteract the quickly decaying model resolution with depth in gravity inversions, different approaches have been developed, such as depth weighting of the regularizer (Li and Oldenburg, 1998) and re-weighted conjugate gradient methods with focusing stabilizers (Zhdanov, 2002). These methods basically operate on the stabilizing functional $\Phi_{m}$ of (16). In contrast, the method proposed here applies a 3D weighting function to the data misfit functional gradient $\nabla \Phi_{d}$. Therefore, the Laplacian stabilizer chosen here is not further altered, and a regularization parameter $\lambda=1$ is used in all following inversion studies.

Before entering the NLCG line-search algorithm, the data functional's gradient is augmented by a depth weighting function $f(z)$ such that the alternated gradient has the form $f(z) \nabla \Phi_{d}$. The weighting function is chosen in a way that the inherently high sensitivities of the model grid cells near the surface, i.e. in the proximity of measurement stations, are damped. Considering the vertical coordinate, where the surface $(z=0)$ defines the upper boundary of the vertical inversion domain, it is desired that $f(z) \approx \alpha<1$ near the surface, and with increasing depth $z>0, f(z) \rightarrow 1$, in order to ease the down-weighting behaviour. Effectively, less weight is thus given to over-sensitive model parameters. A number of preparatory studies, not documented further here, on the undesired effects of this scheme have revealed that the main risk caused by an overly rigorous down-weighting appears to be a premature convergence of the NLCG method to a local minimum. This may be aggravated by noisy data if the signals from deep model parameters are below noise level. However, the scheme was not observed to introduces significant artifacts at depth, 
which can be explained by the fact that the weights of less sensitive parameters at depth are not altered.

While a variety of weighting functions can be considered, the function of (21) was found to deliver the desired damping behaviour. By rewriting (21) to

$$
f(z)=\frac{\alpha+\exp \left[\frac{r}{d z}\left(z-z_{c}\right)\right]}{1+\exp \left[\frac{r}{d z}\left(z-z_{c}\right)\right]},
$$

the depth range over which $f$ changes from $\alpha$ to 1 , and thus the steepness of the function increase, can be chosen by the value of $z_{c}$. An empirical value of $\alpha=0.001$ was found to deliver satisfactory results for the presented inverse problems. Note that $f\left(z=z_{c}\right)=\frac{\alpha+1}{2}$. The choice of $z_{c}$ has to be with respect to the vertical inversion domain, $d z$. Further, the factor $r$ serves as a scaling factor enforcing that $f(z=0) \approx \alpha$.

The depth weighting effect of (17) is demonstrated on the cube model used above, where the anomalous density cube is buried at three different depths ( $\mathrm{Li}$ and Oldenburg, 1998). Figure 2 shows the reproduced cube models, where both vertical gravity data (a-c) and vertical gradiometry data (d-f) was inverted. The corresponding gradient weighting functions are plotted below the inversion results (g-i). For each cube a different value for $z_{c}$ was used, where $z_{c}=\frac{z_{1}+z_{2}}{2}$, and $z_{1}$ and $z_{2}$ are the upper and lower cube boundaries. The vertical inversion domain extends from $z=0 \mathrm{~m}$ to $z=500 \mathrm{~m}(d z=500)$. Also given in each plot title (a-f) is the number of inversion iterations needed to reach a target misfit of $\Phi_{d}=1$.

All inversions recover the anomalies well within the true depths, however with a less exact location of the upper anomaly bounds. One observes a trend to larger depths, which is accompanied, due to equality, by slight overshoots of $\Delta \rho$, with maxima of 1.5 (a), 1.1 (b and c), 1.7 (d and e), and 1.3 (f). Nevertheless, the lower bounds of the reconstructed 
anomalies are well matched, indicating the desired depth focusing behaviour. Carrying trial

runs with values of $z_{c}<\frac{z_{1}+z_{2}}{2}$, yielded inferior results in terms of locating the lower cube boundary.

This basic study indicates that the chosen weighting parameters produce similar results for gravity and gradiometry data types. While not further pursued in this work, it is suggested that the joint inversion of gravity and gradiometry data could involve a datadependent weighting, specifically

$$
f(z) \nabla \Phi_{d}=f_{g_{z}}(z) \nabla \Phi_{g_{z}}+f_{g_{z z}}(z) \nabla \Phi_{g_{z z}}
$$

Defining the weighting behaviour by data type, i.e. $z_{c}^{g_{z}}>z_{c}^{g_{z z}}$, the information contained in the gradiometry data can be activated for shallow structures, while the gravity data contributes to the deeper model resolution.

\section{Inversion parameter constraints}

To restrict potential field data inverse solutions to geologically meaningful ones, it has been recognized that, in addition to using focusing stabilizers, imposing lower and upper bounds on the recovered density contrast is beneficial (Li, 2001). Cardarelli and Fischanger (2006) show that the use of bounding constraints facilitates the inversion of electrical resistivity tomography data, because one becomes less dependent on the time-consuming choice of a proper Lagrange multiplier controlling the influence of a prior model guess. Kim and Kim (2008) also suggest that inverting in a transformed parameter space may produce sharper image rendering if the transformation function enforces tight bounds that are selected from a priori information. This was demonstrated for controlled-source electromagnetic data inversions for bounded electrical conductivity parameters, using the same NLCG inversion 
framework as employed here (Commer and Newman, 2008).

The class of hyperbolic and logarithmic transformation functions typically used in electrical conductivity imaging appear to provide similar benefits for gravity inversion. Such functions map a parameter $m$, bounded by

$$
a<m<b,
$$

with lower and upper bounds $a$ and $b$, to an unbounded domain in the transform space. For the class of parameter transformations employing inverse hyperbolic tangent functions, the transformed parameters $x$ are related to the original density parameters $m$ by

$$
x=f(m, p)=\frac{2}{p} \operatorname{artanh}\left(\frac{2 m-b-a}{b-a}\right) .
$$

The corresponding back-transformation reads

$$
m=\left(\frac{b-a}{2}\right) \tanh \left(\frac{p x}{2}\right)+\frac{b+a}{2} ; \quad-\infty<x<\infty .
$$

Note that because of $\operatorname{artanh}(z)=\frac{1}{2} \ln \left(\frac{1+z}{1-z}\right)$ and by substituting $z=\frac{2 m-b-a}{b-a}$, it can be shown that (Kim and Kim, 2008)

$$
x=f(m, p)=\frac{1}{p} \ln \frac{m-a}{b-m},
$$

which in turn yields an expression equivalent to (19)

$$
m=\frac{a+b \exp (p x)}{1+\exp (p x)} ; \quad-\infty<x<\infty .
$$

Imposing bounds $] a, b[$, positivity or negativity of $m$ can be enforced given adequate prior information. The function parameter $p$ controls the behaviour in the region near $x=0$ of the transformed space, where $m=(a+b) / 2$. While the bounding property has the large benefit of preventing density parameter overshoots, care has to be taken in choosing the function's shape by means of $p$, as shown in the following using the cube model of Figure 2a. 
Figure 3 illustrates $f(m, p)$ for four different values of $p$. In principal, $p$ controls the steepness of $f(m, p)$ in the region where $f$ behaves rather linear. Furthermore, with increasing $p, f$ approaches a step-like function. A step function being discontinuous, it follows that the mapping from $x$ to $m$ becomes unstable for large values of $p$, i.e. $m$ approaches one of the bounds $a$ or $b$ within a short interval of $x$. In the other extreme, $p \rightarrow 0, m$ stays rather invariant towards changes in $x$. The effects of four choices for the parameter $p$ on the cube model are shown in Figure 4. For all four inversions, the bounds were set to $a=-0.5$ and $b=2.5$ in order to have an equal difference between the cube's true density anomaly of 1 $\frac{g}{\mathrm{~cm}^{3}}$ and each bound. Further, the weighting function of Figure $2 \mathrm{~g}$ was employed.

The first inversion (a) was carried out using $p=1.35$, assuming that a transformation function with a slope of approximately one around $x=0$ should account for a well-behaved mapping within a relatively large range of $x$, while still enforcing sufficient influence of the bounding constraints. The corresponding image shows that the true density of $1 \frac{\mathrm{g}}{\mathrm{cm}^{3}}$ is approached within a large area of the true anomaly (outlined by the black box), with the maximum recovered cell anomaly of $\rho=1.5 \frac{\mathrm{g}}{\mathrm{cm}^{3}}$. The next inversion result (b), generated by $p=2$, also leads to a satisfactory image. The reproduction is slightly improved in the upper part of the cube, yet at the expense of a weaker rendering in the bottom part. Here, the maximum anomaly in the image reaches $\rho=1.3 \frac{\mathrm{g}}{\mathrm{cm}^{3}}$. The extreme $p=0.45$ (c) produced an image which only indicates the upper structure of the anomaly. The maximum density contrasts $\left(\rho=0.13 \frac{\mathrm{g}}{\mathrm{cm}^{3}}\right)$ achieved by this inversion are far below the true value, which is in accordance with the function behaviour described above for the limit case $p \rightarrow 0$. It was observed that smaller values of $p$ led to a failure in the NLCG algorithm's line-search procedure. At last, another extreme, $p=4$, was tested. The outcome appears to be satisfying in terms of reproducing the anomaly's shape. The larger density overshoots, with 
a maximum of $\rho=1.7 \frac{\mathrm{g}}{\mathrm{cm}^{3}}$ observed in this result, indicate the increasingly erratic behaviour of $f$ when $p \rightarrow \infty$.

The results confirm that the parameter transformation function employed here is wellbehaved, i.e. approximates a linear mapping within the bounds, as long as the extremes, obviously not recommended, are avoided. All inversions carried out in the following employ a value of $p=1.35$ in the implementation of (18) and (19)

\section{Synthetic subsalt inversion study}

In the following, the gradient weighting scheme is tested on more realistic gravity inversion examples. Synthetic vertical gravity data is produced from a large-scale marine model adapted from a subsalt magnetotelluric imaging study of the Mahogany prospect in the Gulf of Mexico (Newman et al., 2002). The data consists of 405 observation points along diagonal profiles, indicated by dots above the rendered salt structures in the lower Figure 5, and covers an area of approximately $30 \mathrm{~km} \times 30 \mathrm{~km}$. The employed model grid covering the inversion domain has $104 \times 100 \times 70$ cells with a horizontal size of $250 \mathrm{~m}$ and a vertical size of $100 \mathrm{~m}$. In a first simple case, the salt bodies were given a negative density anomaly with respect to a homogeneous host rock. Bathymetry effects and near-surface anomalies, normally of importance in such a case, are not considered here, because it shall be focused on the method's capability of assessing the salt bodies geometry and location. The true host rock density is $2.45 \frac{\mathrm{g}}{\mathrm{cm}^{3}}$, and $2.125 \frac{\mathrm{g}}{\mathrm{cm}^{3}}$ for the salt bodies, with a small random variation $(\approx 1 \%)$ imposed on both. The upper Figure 5 shows the gravity anomalies, calculated with

respect to a reference homogeneous model with a rock density of $2.45 \frac{\mathrm{g}}{\mathrm{cm}^{3}}$. The effect of the salt bodies is obvious in the region between $y=5$ and $y=10 \mathrm{~km}$. A second case involves 
the challenging situation of salt bodies embedded in a layered background with a vertical density gradient from $1.9 \frac{\mathrm{g}}{\mathrm{cm}^{3}}$ below station level to $2.6 \frac{\mathrm{g}}{\mathrm{cm}^{3}}$ at $z=6 \mathrm{~km}$. This gradient causes the salt bodies to have a small negative anomaly below $z=3 \mathrm{~km}$, whereas above, their density blends in with the background. The middle Figure 5 shows the corresponding gravity anomaly.

The two inversion results are shown in Figure 6. The gradient weighting is characterized by a value of $z_{c}=2.5 \mathrm{~km}$ in both cases, and a parameter transformation function with the values $a=-1.0$ and $b=1$ (Equation 18 ) was used. The first inversion result clearly displays both a pro and con of the method. On the one hand, both location and extent of the salt bodies is recovered to a fairly good degree (Figure 6a), without using a starting model with prior information. On the other hand, the model smoothing constraints produce a rather diffuse image. The second inversion basically reconstructs the vertical density gradient of the host rock. The gravity map of this scenario (middle Figure 5) already indicated a de facto positive density anomaly in the center region. Figure $6 \mathrm{~b}$ shows that the inversion tries to recover this situation by concentrating slightly more mass in the center region (middle slice).

\section{Field data inversion}

Finally, a field data inversion carried out earlier by Li and Oldenburg (1998) is repeated here for demonstrating the usefulness of the gradient weighting approach. The reader is referred to their article for more geological details about the survey. The vertical gravity data was collected at Heath Steele Mines, a copper, lead and zinc mine (operation closed in 1999) in northern New Brunswick (Canada). Figure 7 (upper) contours the Bouguer anomalies, 
which comprise 443 stations depicted by the dots on the surface $(z=0)$ of the model volume (lower). The maximum positive anomaly corresponds to a massive sulphide ore body embedded in gabbroic intrusions. This inversion used the transformation parameters $a=-1, b=1$, a gradient weighting defined by $z_{c}=0.2 \mathrm{~km}$, and a grid of size $50 \times 50 \times 20$ with $25 \mathrm{~m}$ cell size to cover the $1 \mathrm{~km} \times 1 \mathrm{~km}$ survey area. Figure 8 shows three cross-sections of the reconstructed density. The large positive anomaly in the upper section pertains to a large meta-gabbro intrusion, which appears to be connected (middle section) to the sulphide body to the east (lower section). This spatial distribution becomes also clear in the lower part of Figure 7, where the reproduced density anomalies exceeding $0.3 \frac{\mathrm{g}}{\mathrm{cm}^{3}}$ are rendered. No significant negative densities were produced by the inversion, although negativity was enabled by the chosen bounds. The result demonstrates that a relatively detailed model, suitable for subsequent studies, can be obtained without a prior model.

\section{CONCLUSIONS}

A method for the inversion of gravity data using a minimum amount of prior information has been presented. It is often favorable to reconstruct compact gravity sources by allowing sharp density boundaries in an inversion. On the other hand, a rather unconstrained method has the advantage of an unbiased model assessment in the presence of uncertain prior information. Moreover, the method presented here is proposed for a joint inversion framework for gravity and other geophysical data. Rectangular meshes provide a flexible way of incorporating equality constraints for structural resemblance in complex models between different geophysical attributes which are not joined through the different data types. While the hypothesis of structural resemblance may not hold for all types of attributes, the joint inversion of electrical/electromagnetic data for example, sensitive to electrical con- 
ductivity anomalies due to fluids, with gravity data has the potential of producing less ambiguous reservoir (hydrocarbon or geothermal) images.

The class of hyperbolic parameter transformation functions employed here appear favorable for gravity inversions since positivity or negativity can be enforced, if one has such prior knowledge. The synthetic study illustrated that it is preferable to keep a well-behaved (linear) mapping between parameter space and transformed space, in order to reduce the risk for parameter over- or undershoots in an inversion. Weighting the data gradient of the objective functional provides a straightforward way of counteracting the natural decay of the gravity kernels with depth. Using a uniform model regularization operator, the only parameters are given by the degree and steepness of the weighting function, i.e. $\alpha$ and $\mathrm{zc}$ in (17). Similar to cooling methods used for regularization parameter assessment, a series of inversions approaching a desired target misfit could be used for refinement of the weighting parameter $\mathrm{z}_{\mathrm{c}}$. The method does not prohibit reconstruction of near-surface gravity sources per se. Suggested here is a "sweep"-approach involving multiple inversion steps. A first step, focusing on deeper model regions, provides the starting model for subsequent inversion sweeps, with their focus regions moving towards the surface by decreasing $\mathrm{z}_{\mathrm{c}}$.

\section{ACKNOWLEDGMENTS}

This work was partially supported by the U.S. Department of Energy and LBNL under Contract No. DE-AC02-05CH11231. I would also like to thank Michael Hoversten and Betty A. Johnson from Chevron Energy Technology Co. for advice on the subsalt study. Special thanks to Yaoguo Li and DouglasW.Oldenburg for providing their field data inversion input. 


\section{APPENDIX}

\section{Singularities for $g_{\ell \ell^{\prime}}$}

For the off-diagonal tensor gradient components $g_{\ell \ell^{\prime}}$, singularities are encountered when the argument $c_{k}+r_{i j k}$ of the logarithmic function of (15) becomes zero. This happens when both $c_{k} \rightarrow 0$ and $r_{i j k} \rightarrow 0$, i.e. an observation point approaches one cube corner $(i, j, k)$. A method proposed by Pohánka (1988) for his formulation developed for a polyhedral body in principal adds a small quantity $\varepsilon$ to the argument of the logarithm, where $\varepsilon$ is chosen such that it is very small relative to the polyhedron's characteristic dimension. The singularity also occurs when, for a given cell corner $(i, j, k)$ and coordinate orientation corresponding to $k, c_{k}=-r_{i j k}$. If a field point is right above the corner (assuming here that $k$ refers to the vertical), the logarithm in (15) becomes unbounded due to $a_{i}^{2}+b_{j}^{2}=\varepsilon$ with $\varepsilon \ll 1$ and $r_{i j k} \rightarrow\left|c_{k}\right|$ for $\varepsilon \rightarrow 0$. For this singularity case, an alternative to Pohánka's method is proposed, because it also reduces the number of logarithms to be calculated. Taking the exponential of the summation part of equation (15), one obtains

$$
\begin{aligned}
\tilde{g}_{\ell \ell^{\prime}} & =\exp \left[\sum_{i=1}^{2} s_{i} \sum_{j=1}^{2} s_{j} \sum_{k=1}^{2} s_{k} \ln \left(c_{k}+r_{i j k}\right)\right] \\
& =\prod_{i=1}^{2} \prod_{j=1}^{2} \prod_{k=1}^{2} \exp \left[s_{i} s_{j} s_{k} \ln \left(c_{k}+r_{i j k}\right)\right]
\end{aligned}
$$

For a fixed pair $(i, j)$ the product $s_{i} s_{j} s_{k}$ always has a different sign for $k=1$ and $k=2$. Assuming for example, that the argument of the exponential function is positive/negative for $k=1 / k=2$, the product of $\tilde{g}_{\ell \ell^{\prime}}$ over $k$ can be further rewritten to

$$
\prod_{k=1}^{2} \exp \left[s_{i} s_{j} s_{k} \ln \left(c_{k}+r_{i j k}\right)\right]=\frac{r_{i j 1}-\left|c_{1}\right|}{r_{i j 2}-\left|c_{2}\right|}
$$


The geometrical relationship between the vector lengths in equation (A-2) is illustrated in Figure 9. For the shown geometries it can be seen that

$$
\frac{r_{i j 1}-\left|c_{1}\right|}{r_{i j 2}-\left|c_{2}\right|}=\frac{\left|c_{1}\right|\left(\frac{1}{\cos \alpha_{1}}-1\right)}{\left|c_{2}\right|\left(\frac{1}{\cos \alpha_{2}}-1\right)}
$$

The singularity caused by $\alpha_{2} \rightarrow 0$ in the denominator, as $\mathbf{r}$ approaches the corner, can be removed by substituting each term within parentheses by its second order Taylor series, specifically

$$
\frac{1}{\cos \alpha}-1 \approx \frac{\alpha^{2}}{2}
$$

for $\alpha \ll 1$. Moreover, one has for the two arc lengths $\left|c_{1}\right| \alpha_{1}$ and $\left|c_{2}\right| \alpha_{2}$

$$
\left|c_{1}\right| \alpha_{1} \approx\left|c_{2}\right| \alpha_{2}
$$

Applying both these approximations yields

$$
\frac{r_{i j 1}-\left|c_{1}\right|}{r_{i j 2}-\left|c_{2}\right|} \approx \frac{\left|c_{1}\right|}{\left|c_{2}\right|} \frac{\alpha_{1}^{2}}{\alpha_{2}^{2}} \approx \frac{\left|c_{2}\right|}{\left|c_{1}\right|} .
$$

The exact representation given by the left hand side in (A-3) can be used as long as both enumerator and denominator are representable by the given machine precision. In practice, it is recommended to base the criterion for switching between the exact representation and its approximation, $\frac{\left|c_{2}\right|}{\left|c_{1}\right|}$, on a threshold value for $\varepsilon=a_{i}^{2}+b_{j}^{2}$. The validity of the approximation depends on the length of $c_{1}$ (Figure 9). Using the standard cubic model, Figure 10 compares the left hand side of (A-3), with the corresponding approximations $\frac{\left|c_{2}\right|}{\left|c_{1}\right|}$, shown as horizontal lines, for three different values of $c_{1}$. All lengths are given in fractions of a prism cell size of $\Delta=20 \mathrm{~m}$. The results show that the ratio approaches the value $\frac{\left|c_{2}\right|}{c_{1} \mid}$ when $\varepsilon<0.1\left|c_{1}\right|$. The difference between the left and right hand side of equation A-3 quickly decreases with $\varepsilon$. The annotated percentages in Figure 10 denote the percentage difference for a value of $\varepsilon=10^{-7}$. 
To conclude, the number of logarithmic functions in equation (15) is reduced in the alternative form

$$
g_{\ell \ell^{\prime}}=-\gamma \varrho \ln \left(\prod_{i=1}^{2} \prod_{j=1}^{2} \frac{r_{i j 1}-\left|c_{1}\right|}{r_{i j 2}-\left|c_{2}\right|}\right) .
$$

A potential singularity for a given field point and the prism corner $(i, j, k)$ where $\varepsilon=$ $a_{i}^{2}+b_{j}^{2} \rightarrow 0\left(r_{i j k} \rightarrow\left|c_{k}\right|\right)$, is removed by approximating the factor $\frac{r_{i j 1}-\left|c_{1}\right|}{r_{i j 2}-\left|c_{2}\right|}$ by the factor $\frac{\left|c_{2}\right|}{\left|c_{1}\right|}$. It is suggested to base a criterion for switching between the two factors by evaluating the distance $\left|c_{k}\right|$. Using the standard cube model, it was calculated that the error due to the approximation is below one percent if $\varepsilon<0.1\left|c_{k}\right|$. 


\section{REFERENCES}

Bosch, M., R. Meza, R. Jiménez, and A. Hönig, 2006, Joint gravity and magnetic inversion in 3d using monte carlo methods: Geophysics, 71, G153-G156.

Boulanger, O., and M. Chouteau, 2001, Constraints in 3D gravity inversion: Geophys. Prosp., 49, 265-280.

Camacho, A. G., F. G. Montesinos, and R. Vieira, 2000, Gravity inversion by means of growing bodies: Geophysics, 65, 95-101.

Cardarelli, E., and F. Fischanger, 2006, 2d data modelling by electrical resistivity tomography for complex subsurface geology: Geophysical Prospecting, 54, 121-133.

Commer, M., and G. Newman, 2008, New advances in three-dimensional controlled-source electromagnetic inversion: Geophysical Journal International, 172, 513-535.

Dias, F. J. S. S., V. C. F. Barbosa, and J. ao B. C. Silva, 2009, 3d gravity inversion through an adaptive-learning procedure: Geophysics, 74, I9-I21.

Farquharson, C., and D. Oldenburg, 2004, A comparison of automatic techniques for estimating the regularization parameter in non-linear inverse problems: Geophysical Journal International, 156, 411-425.

Forsberg, R., 1984, A study of terrain corrections, density anomalies, and geophysical inversion methods in gravity field modeling: Report 355, Department of Geodetic Science and Surveying, The Ohio State University.

Fregoso, E., and L. Gallardo, 2006, Cross-gradients joint 3d inversion with applications to gravity and magnetic data: Geophysics, 74, L31-L42.

Holstein, H., and B. Ketteridge, 1996, Gravimetric analysis of uniform polyhedra: Geophysics, 61, 357-364.

Holstein, H., P. Schurholz, A. J. Starr, and M. Chakraborty, 1999, Comparison of gravi- 
metric formulas for uniform polyhedra: Geophysics, 64, 1438-1446.

Kim, H., and Y. Kim, 2008, Lower and upper bounding constraints of model parameters in inversion of geophysical data: 78th Ann. Internat. Mtg, Soc. of Expl. Geophys., 692-696.

Last, B. J. J., and K. Kubik, 1983, Compact gravity inversion: Geophysics, 48, 713-721.

Li, X., and M. Chouteau, 1998, Three-dimensional gravity modeling in all space: Surveys in Geophysics, 19, 339-368.

Li, Y., 2001, 3D inversion of gravity gradiometer data: 71st Ann. Internat. Mtg, Soc. of Expl. Geophys., 1470-1473.

Li, Y., and D. Oldenburg, 2003, Fast inversion of large-scale magnetic data using wavelet transforms and a logarithmic barrier method: Geophysical Journal International, 152, $251-265$.

Li, Y., and D. W. Oldenburg, 1998, 3-D inversion of gravity data: Geophysics, 63, 109-119.

Nagy, D., 1966, The gravitational attraction of a right rectangular prism: Geophysics, 31, $362-371$.

Newman, G., and D. Alumbaugh, 1997, Three-dimensional massively parallel electromagnetic inversion- i. theory: Geophysical Journal International, 128, 345-354.

Newman, G., G. Hoversten, and D. Alumbaugh, 2002, Three-dimensional magnetotulleric modeling and inversion: application to sub-salt imaging: Three-Dimensional Electromagnetics, Proc. Sec. Int. Symp., Elsevier, Amsterdam, 127-152.

Okabe, M., 1979, Analytical expressions for gravity anomalies due to homogeneous polyhedral bodies and translations into magnetic anomalies: Geophysics, 44, 730-741.

Oldenburg, D. W., 1974, The inversion and interpretation of gravity anomalies: Geophysics, 39, 526-536.

Pedersen, L., 1977, Interpretation of potential field data - a generalized inverse approach: 
Geophysical Prospecting, 25, 199-230.

Plouff, D., 1976, Gravity and magnetic-fields of polygonal prisms and application to magnetic terrain corrections: Geophysics, 41, 727-741. (Erratum in GEO-54-9-1213-1213; Discussion in GEO-54-9-1213-1213 with reply by author).

Pohánka, V., 1988, Optimum expression for computation of the gravity field of a homogeneous polyhedral body: Geophysical Prospecting, 36, 733-751.

Rene, R. M., 1986, Gravity inversion using open reject and shape-of-anomaly fill criteria: Geophysics, 51, 988-994.

Silva, J., and V. Barbosa, 2006, Interactive gravity inversion: Geophysics, 71, J1-J9.

Zhdanov, M., 1988, Integral transforms in geophysics: Springer Verlag.

—, 2002 , Geophysical inverse theory and regularization problems: Elsevier, Amsterdam.

Zhdanov, M., R. Ellis, and S. Mukherjee, 2004, Three-dimensional regularized focusing inversion of gravity gradient tensor component data: Geophysics, 69, 925-937.

Zidarov, D., and Z. Zhelev, 1970, On obtaining a family of bodies with identical exterior fields - Method of bubbling: Geophys. Prosp., 18, 14-33. 


\section{LIST OF FIGURES}

1 The 3 -D gravity effect in $\mu \mathrm{Gal}$ for the gravity components $g_{z}$ (a) and the gradiometry components $g_{z z}$ (b) and $g_{x y}$ (c) calculated for the standard cubic model of Li and Chouteau (1998) using equations (10), (14), and (15), respectively. The cube of size $20 \mathrm{~m} \times 20 \mathrm{~m} \times 20 \mathrm{~m}$ (corners are at $\pm 10 \mathrm{~m}$ ) has the (unit) density of $\rho=1.0 \frac{\mathrm{g}}{\mathrm{cm}^{3}}$. The dots in the plane $z=0$ (a), along the diagonals in (b) and in the planes through $x=0$ and $y=0$ (c) indicate the regions where the gravity/gradiometry effect is zero. The calculated ranges of values are $[-24.07,38.43]$ in (b) and $[-84.80,84.80]$ in (c). Note that for better visibility only the range $[-15,15]$ is shown in $(\mathrm{c})$. The subplots (d-f) depict the numerical error caused by using the approximating formulae (6), (7), and (8).

2 Gravity (a-c) and gradiometry (d-f) data produced from a cube with anomalous density at three different depths are inverted using weighting functions (g-i) applied to the gradient of the data objective functional. The true geometry of the cubic anomaly is outlined by the black lines. The anomaly top is at (in $\mathrm{m}$ ) $z_{1}=50, z_{1}=100$, and $z_{1}=150$, for cube 1,2 , and 3 , respectively; the anomaly bottom is $z_{2}=z_{1}+200$. The number of inversion iterations carried out to reach $\Phi_{d}=1$ is given in each plot title of (a-f).

3 Graph of four different hyperbolic parameter transformation functions characterized by the parameter $p$ in equation (18).

4 The effect due to different parameter transformation functions. The effect of the parameter $p$ in (18) is shown here for four different values. All inversions used a similar gradient weighting scheme as employed for the results of Figure 2

$5 \quad$ The synthetic gravity data field $g_{z}$ created from a model from Newman et al. (2002) for the Mahogany prospect (Gulf of Mexico), where electrically resisitive salt structures were given densities of $2.125 \frac{\mathrm{g}}{\mathrm{cm}^{3}}$. The first inversion study involves a homogeneous 
background density of $2.45 \frac{\mathrm{g}}{\mathrm{cm}^{3}}$, producing the gravity anomalies shown in the upper figure. The anomalies below are produced from a background with a vertical density increase from 1.9 to $2.6 \frac{\mathrm{g}}{\mathrm{cm}^{3}}$. The salt bodies are rendered in the lower figure, with the 405 measurement locations indicated by the dots at $z=0$.

6 Inversion of the $g_{z}$ data shown in Figure 5 using gradient depth weighting. (a) Inversion of data produced from the salt bodies embedded in a homogeneous background. Rendered are anomalies with inversion grid cell values $\Delta \rho<-0.05 \frac{\mathrm{g}}{\mathrm{cm}^{3}}$. (b) Inversion of data produced from a background with vertical density increase, causing the salt bodies to be partly hidden.

$7 \quad$ Vertical gravity data from a survey at Heath Steele Mines (New Brunswick), and inversion result. Shown are the Bouguer anomalies produced by intrusive mafic igneous rocks containing a sulphide ore body. The contours in the lower figure display reconstructed density anomalies $\Delta \rho>0.3 \frac{\mathrm{g}}{\mathrm{cm}^{3}}$, with a maximum of $0.51 \frac{\mathrm{g}}{\mathrm{cm}^{3}}$. The three dotted lines in the Bouguer anomaly map indicate the positions of three sections analyzed by $\mathrm{Li}$ and Oldenburg (1998).

8 Cross-sections from the Heath Steele Mines data inversion corresponding to the three profile lines in the upper Figure 7. The sections exhibit similar features as observed by Li and Oldenburg (1998). Their UTM Easting coordinates are given for reference in each plot title.

9 Computation of $g_{\ell \ell^{\prime}}$ : Illustration of the geometrical relationships used for approximating unbounded expressions in the formula for $g_{\ell \ell^{\prime}}$ by a bounded term for the case $a_{i}^{2}+b_{j}^{2}=\varepsilon \ll 1$.

10 Computation of $g_{\ell \ell^{\prime}}$ : Plot of the ratio $r=\frac{r_{i j 1}-\left|c_{1}\right|}{r_{i j 2}-\left|c_{2}\right|}$ against $\varepsilon=a_{i}^{2}+b_{j}^{2}$ for the three different values $\left|c_{1}\right|=10^{-4} \Delta_{z}, 10^{-5} \Delta_{z}, 10^{-6} \Delta_{z}\left(\Delta_{z}=\right.$ vertical cell size). With decreasing $\varepsilon$, 
$r$ converges to the approximation (straight lines) given by (A-3). 

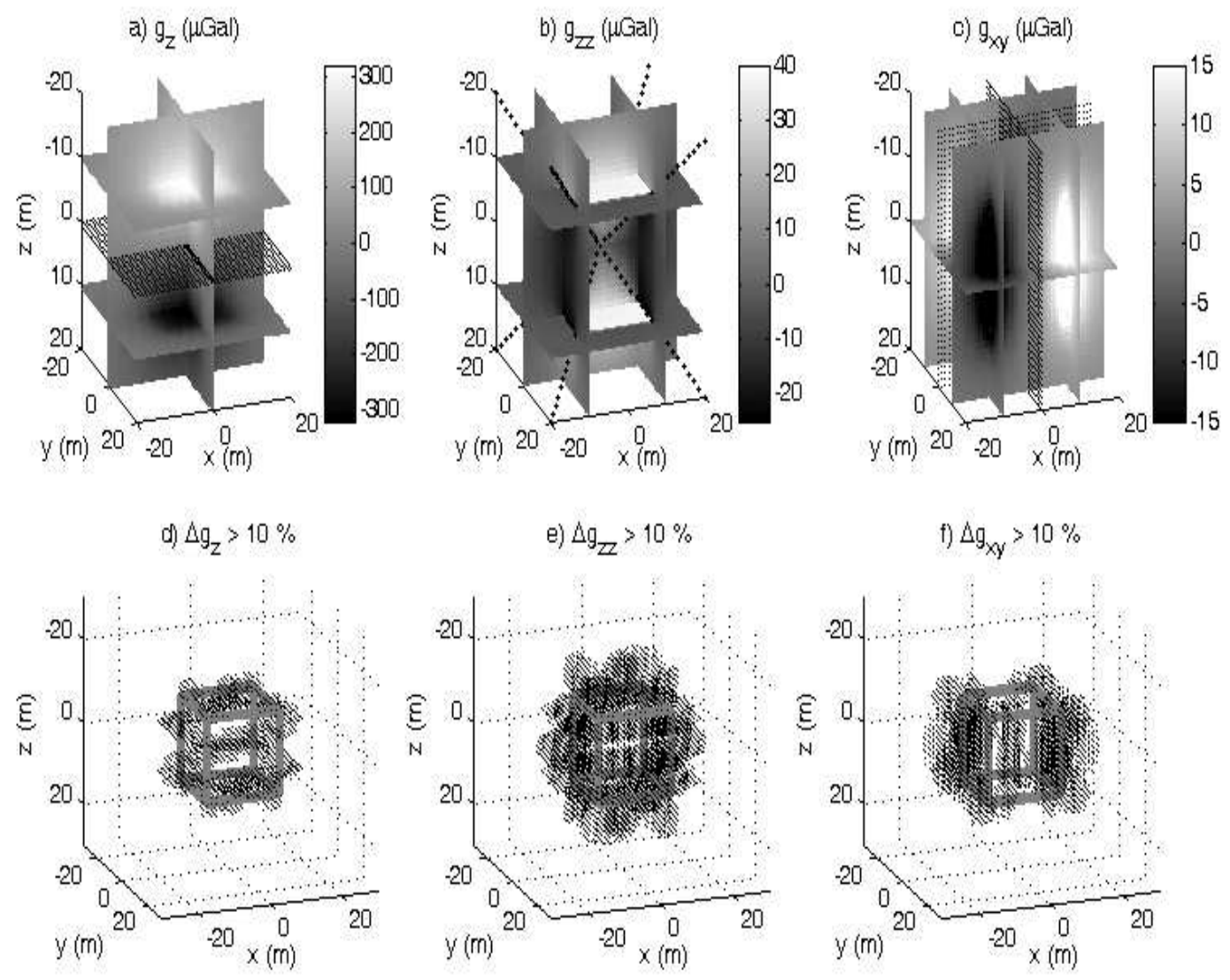

Figure 1: The 3-D gravity effect in $\mu \mathrm{Gal}$ for the gravity components $g_{z}$ (a) and the gradiometry components $g_{z z}$ (b) and $g_{x y}$ (c) calculated for the standard cubic model of Li and Chouteau (1998) using equations (10), (14), and (15), respectively. The cube of size $20 \mathrm{~m} \times 20 \mathrm{~m} \times 20 \mathrm{~m}($ corners are at $\pm 10 \mathrm{~m})$ has the (unit) density of $\rho=1.0 \frac{\mathrm{g}}{\mathrm{cm}^{3}}$. The dots in the plane $z=0(\mathrm{a})$, along the diagonals in (b) and in the planes through $x=0$ and $y=0$ (c) indicate the regions where the gravity/gradiometry effect is zero. The calculated ranges of values are $[-24.07,38.43]$ in (b) and $[-84.80,84.80]$ in (c). Note that for better visibility only the range $[-15,15]$ is shown in $(\mathrm{c})$. The subplots (d-f) depict the numerical error caused by using the approximating formulae (6), (7), and (8). 
a) $g_{z}$ for cube 1 , iterations: 22

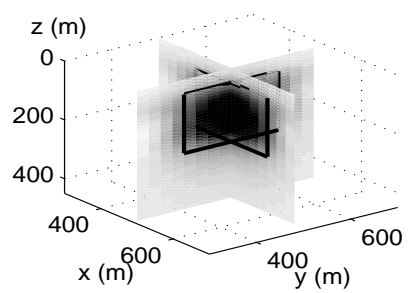

d) $g_{z z}$ for cube 1, iterations: 90

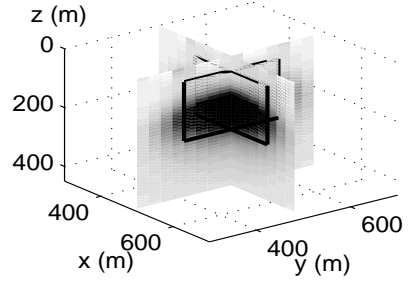

g) cube 1: $z_{c}=150, \alpha=0.001$

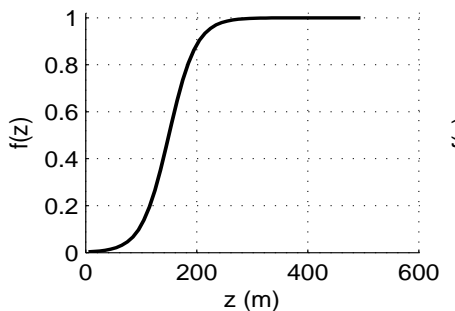

b) $g_{z}$ for cube 2, iterations: 21

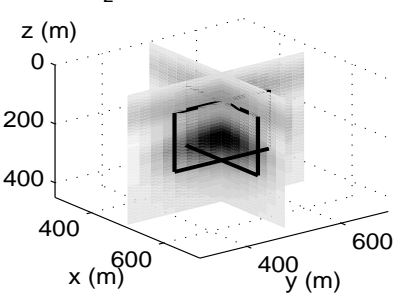

e) $g_{z z}$ for cube 2 , iterations: 88

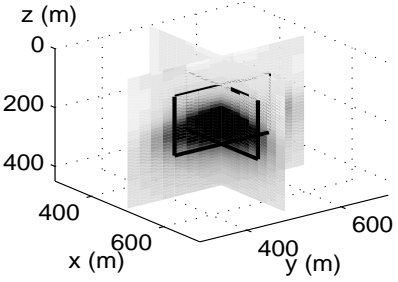

h) cube 2: $z_{c}=200, \alpha=0.001$

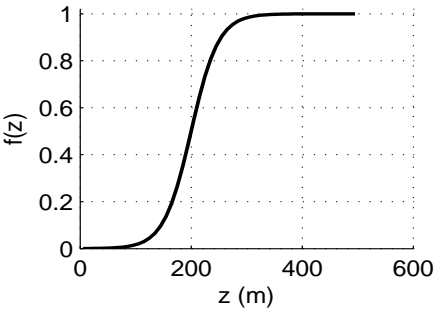

c) $g_{z}$ for cube 3 , iterations: 30

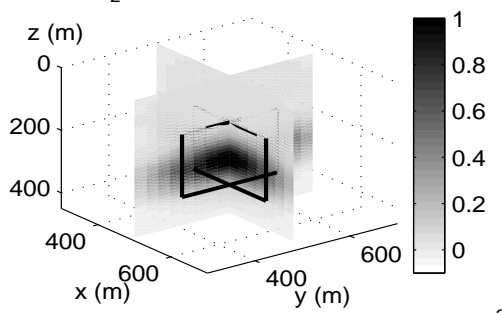

f) $g_{z z}$ for cube 3 , iterations: $58^{\Delta \rho\left(\mathrm{g} / \mathrm{cm}^{3}\right)}$

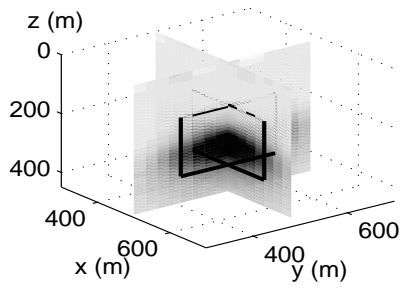

i) cube 3: $z_{C}=250, \alpha=0.001$

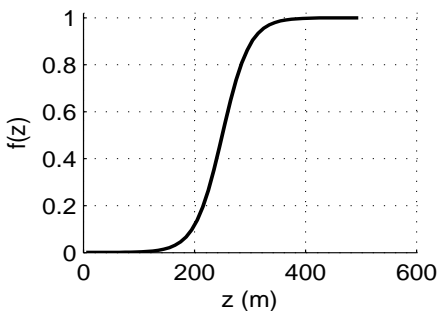

Figure 2: Gravity (a-c) and gradiometry (d-f) data produced from a cube with anomalous density at three different depths are inverted using weighting functions (g-i) applied to the gradient of the data objective functional. The true geometry of the cubic anomaly is outlined by the black lines. The anomaly top is at (in m) $z_{1}=50, z_{1}=100$, and $z_{1}=150$, for cube 1,2 , and 3 , respectively; the anomaly bottom is $z_{2}=z_{1}+200$. The number of inversion iterations carried out to reach $\Phi_{d}=1$ is given in each plot title of (a-f). 


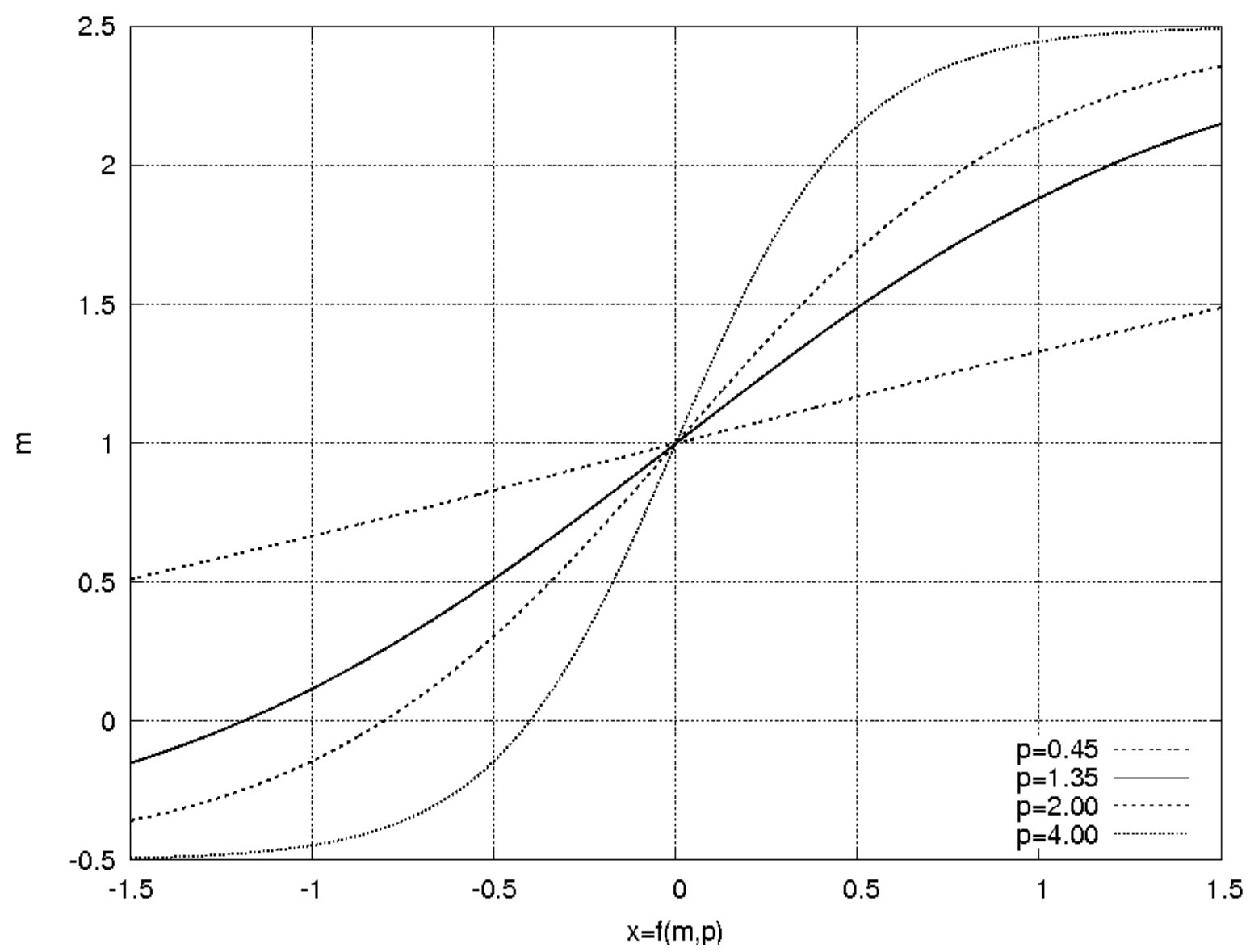

Figure 3: Graph of four different hyperbolic parameter transformation functions characterized by the parameter $p$ in equation (18). 

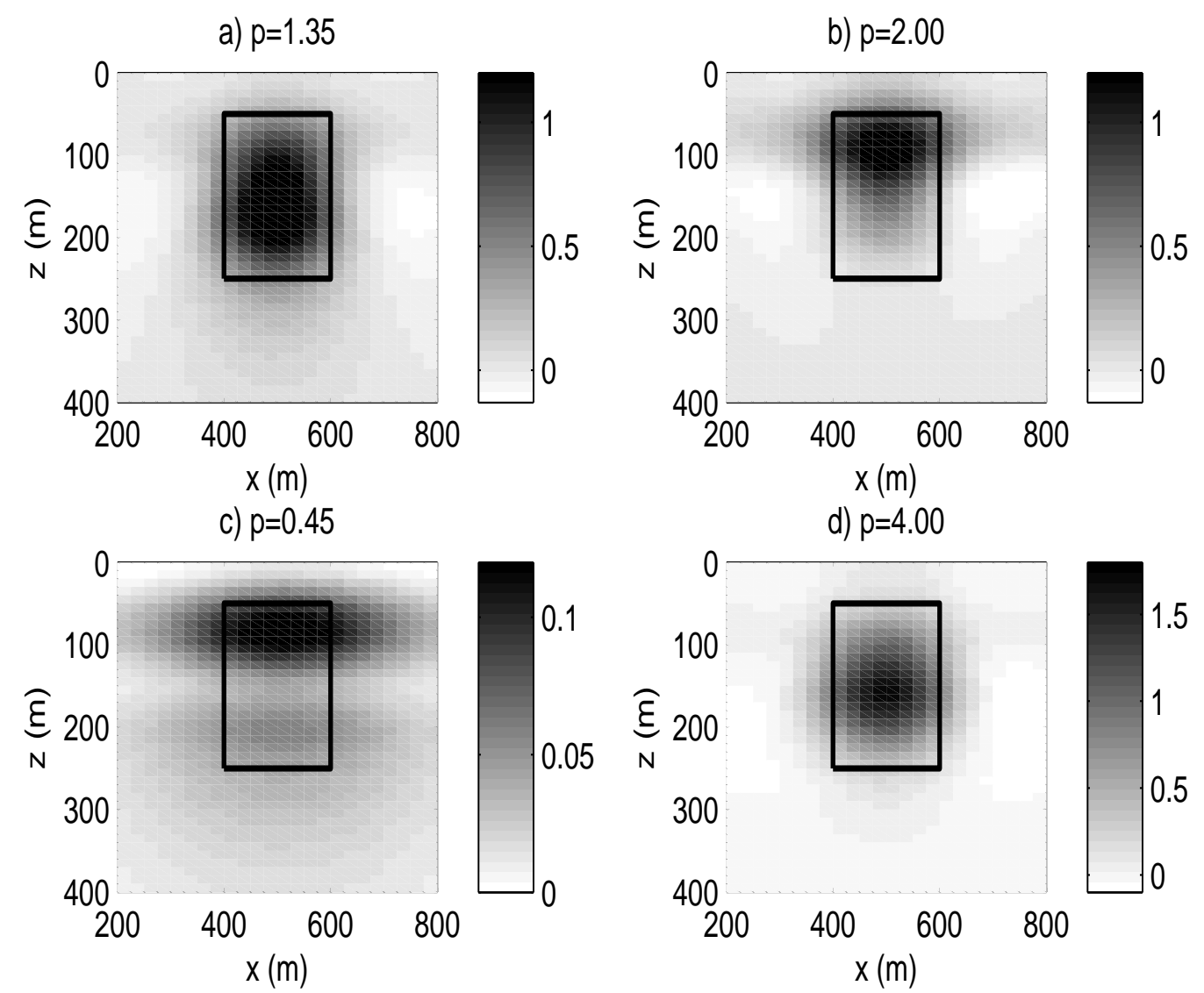

Figure 4: The effect due to different parameter transformation functions. The effect of the parameter $p$ in (18) is shown here for four different values. All inversions used a similar gradient weighting scheme as employed for the results of Figure 2 


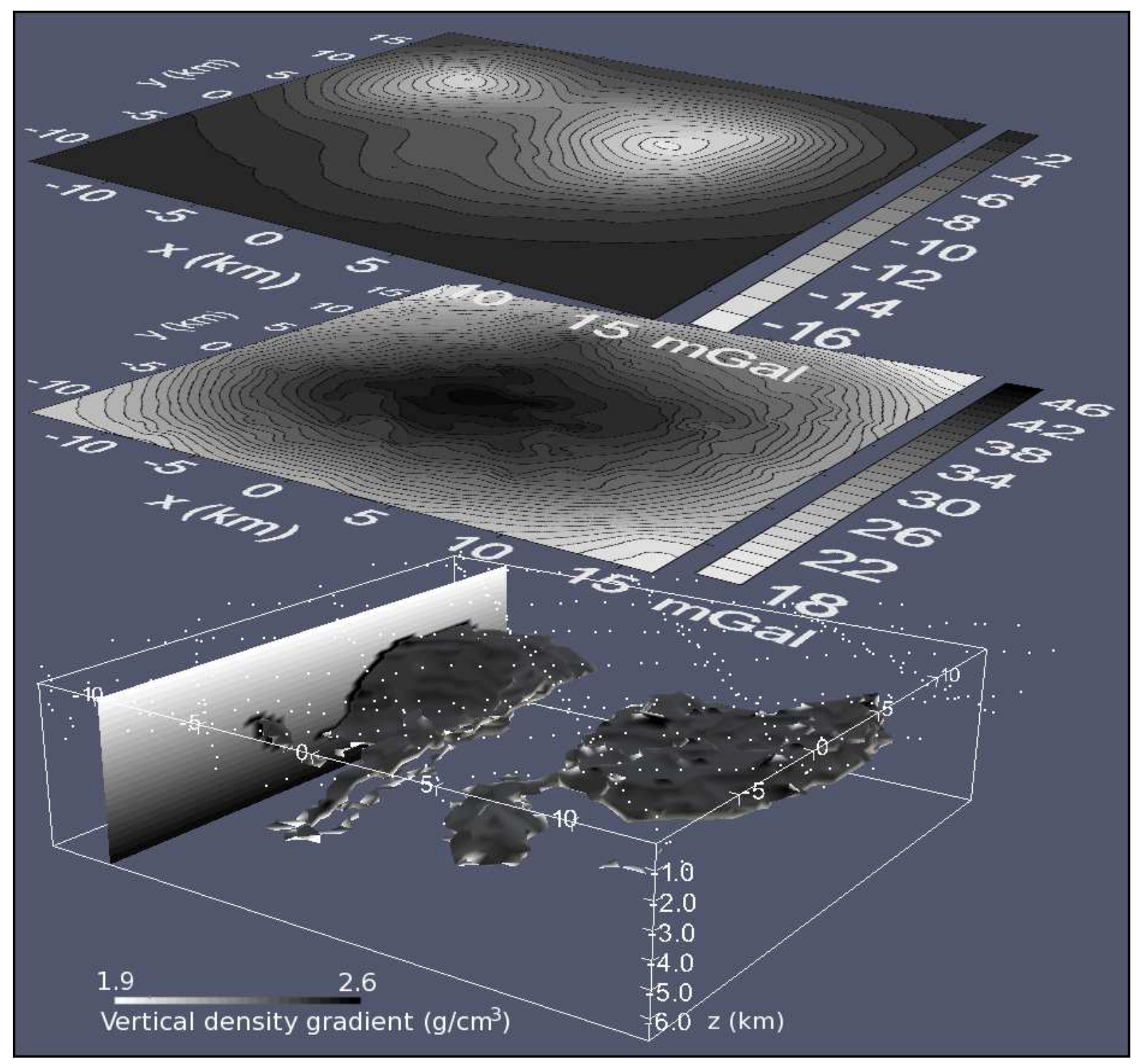

Figure 5: The synthetic gravity data field $g_{z}$ created from a model from Newman et al. (2002) for the Mahogany prospect (Gulf of Mexico), where electrically resisitive salt structures were given densities of $2.125 \frac{\mathrm{g}}{\mathrm{cm}^{3}}$. The first inversion study involves a homogeneous background density of $2.45 \frac{\mathrm{g}}{\mathrm{cm}^{3}}$, producing the gravity anomalies shown in the upper figure. The anomalies below are produced from a background with a vertical density increase from 1.9 to $2.6 \frac{\mathrm{g}}{\mathrm{cm}^{3}}$. The salt bodies are rendered in the lower figure, with the 405 measurement locations indicated by the dots at $z=0$. 


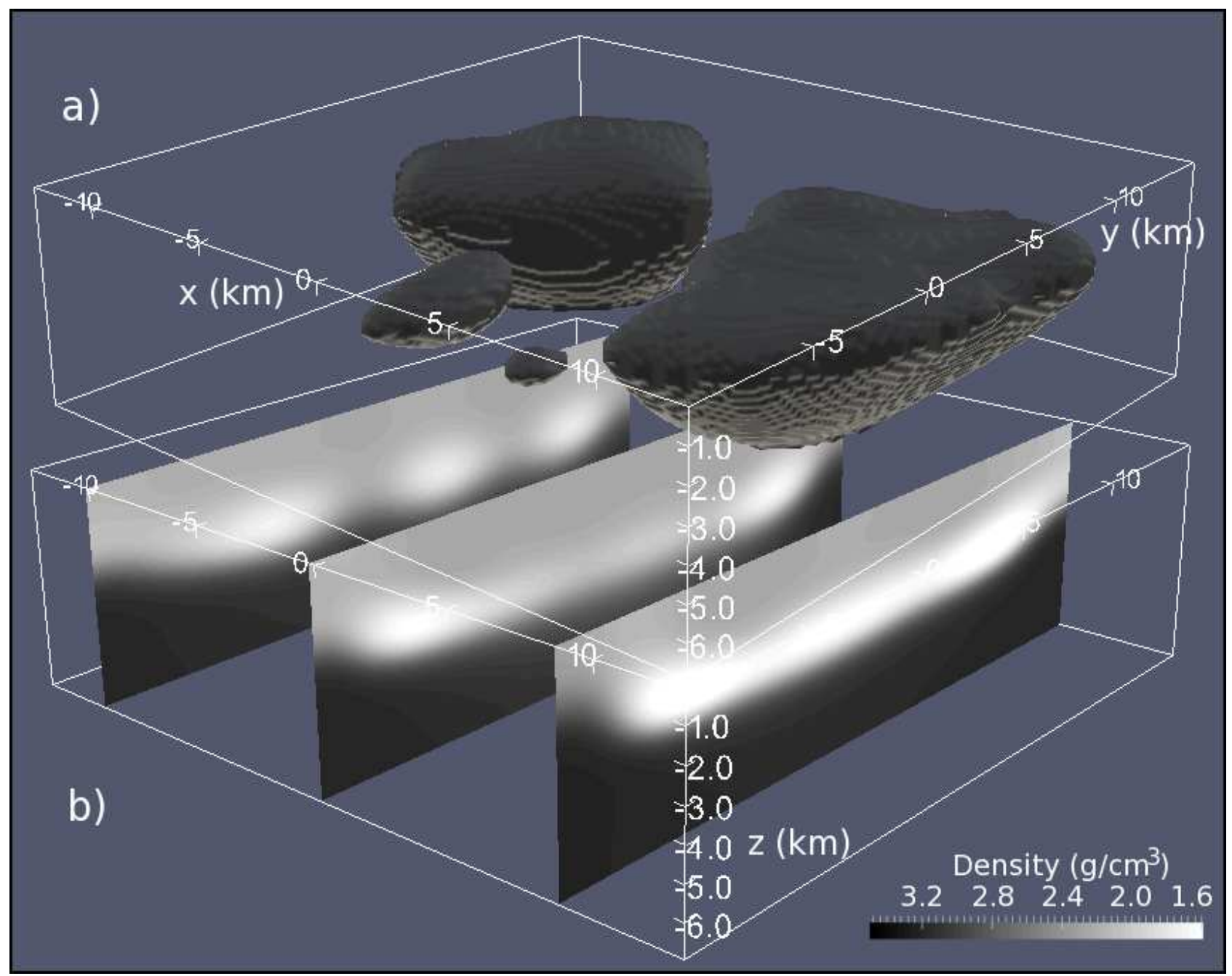

Figure 6: Inversion of the $g_{z}$ data shown in Figure 5 using gradient depth weighting. (a) Inversion of data produced from the salt bodies embedded in a homogeneous background. Rendered are anomalies with inversion grid cell values $\Delta \rho<-0.05 \frac{\mathrm{g}}{\mathrm{cm}^{3}}$. (b) Inversion of data produced from a background with vertical density increase, causing the salt bodies to be partly hidden. 


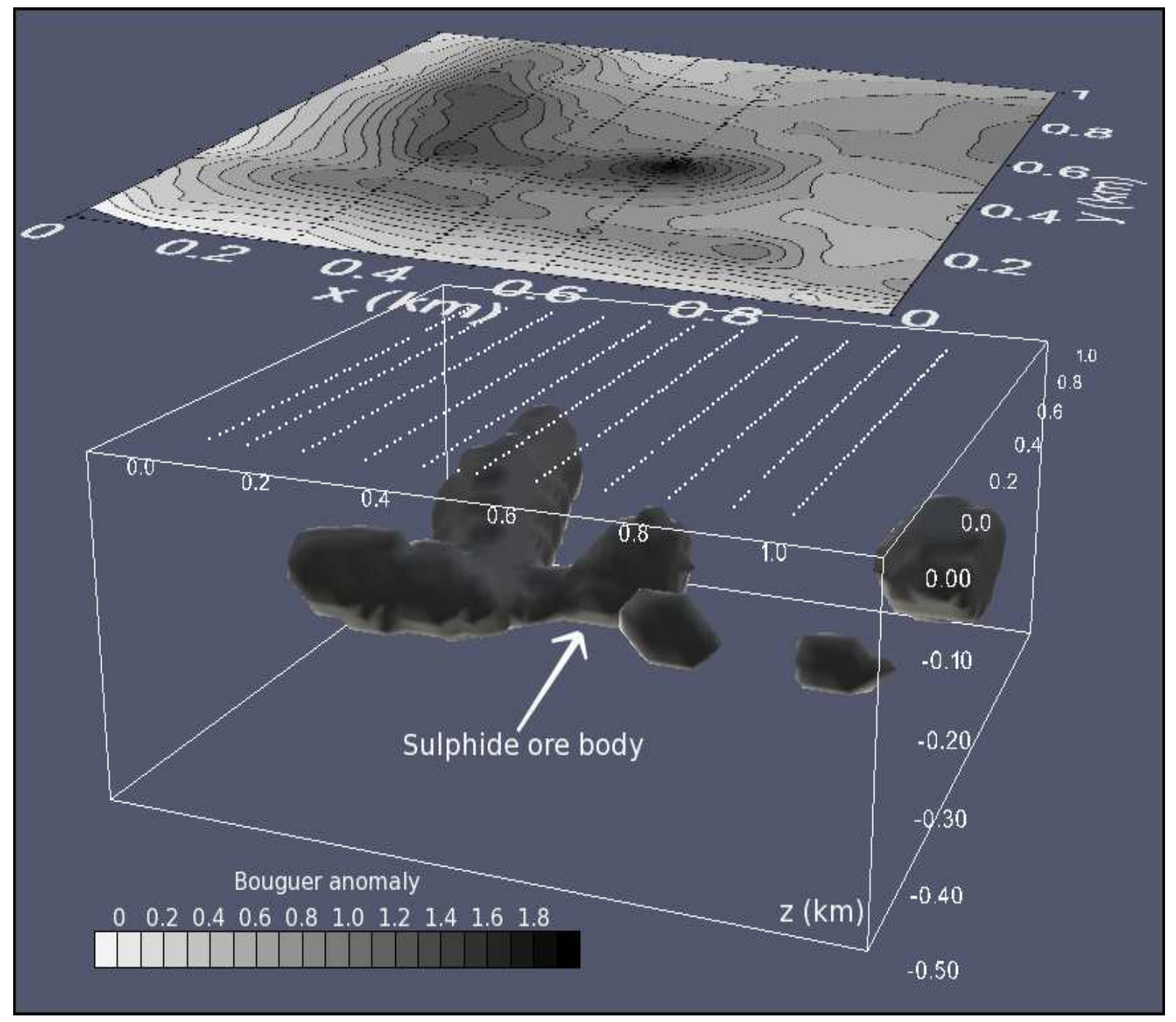

Figure 7: Vertical gravity data from a survey at Heath Steele Mines (New Brunswick), and inversion result. Shown are the Bouguer anomalies produced by intrusive mafic igneous rocks containing a sulphide ore body. The contours in the lower figure display reconstructed density anomalies $\Delta \rho>0.3 \frac{\mathrm{g}}{\mathrm{cm}^{3}}$, with a maximum of $0.51 \frac{\mathrm{g}}{\mathrm{cm}^{3}}$. The three dotted lines in the Bouguer anomaly map indicate the positions of three sections analyzed by Li and Oldenburg (1998). 


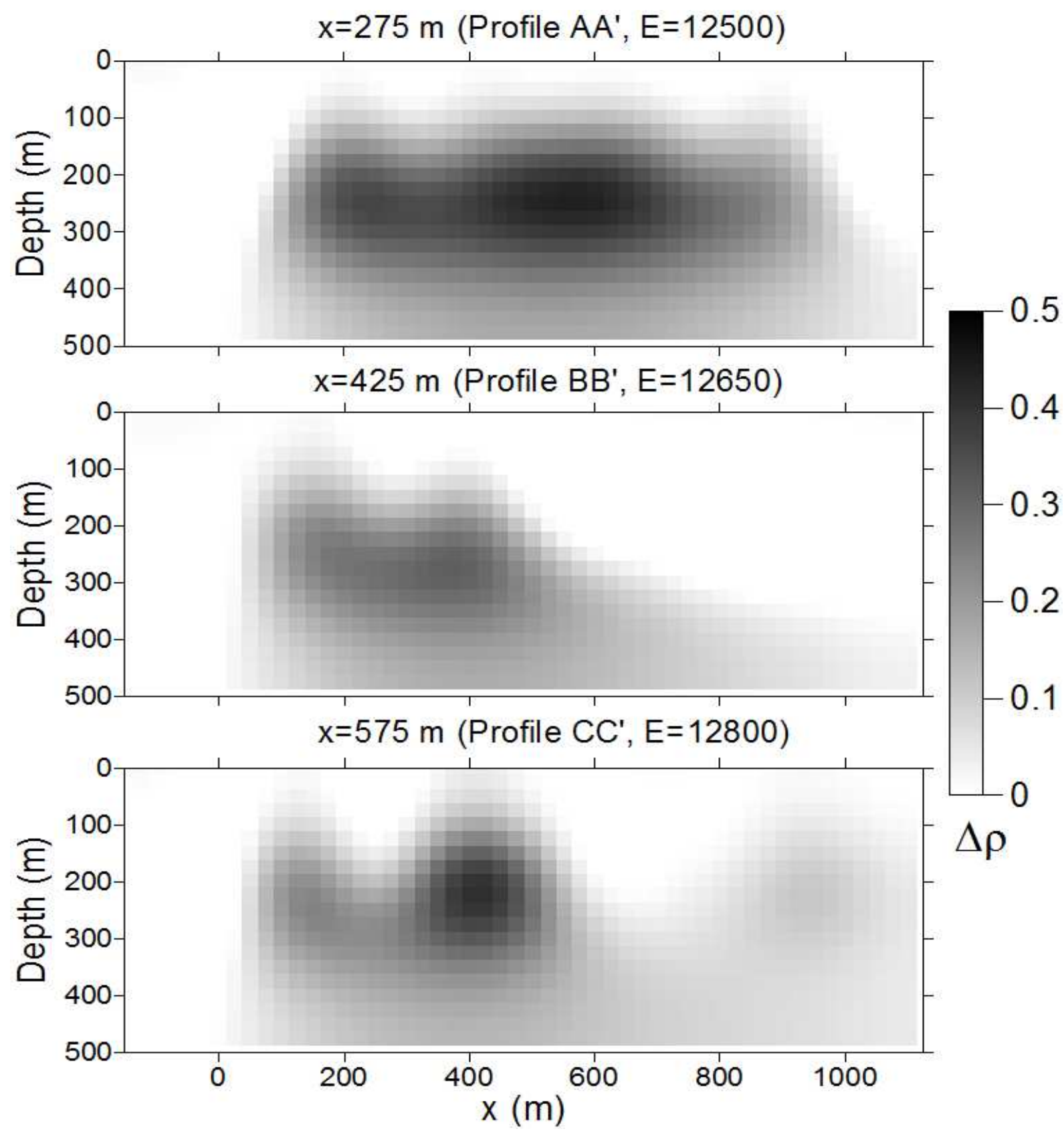

Figure 8: Cross-sections from the Heath Steele Mines data inversion corresponding to the three profile lines in the upper Figure 7. The sections exhibit similar features as observed by Li and Oldenburg (1998). Their UTM Easting coordinates are given for reference in each plot title. 


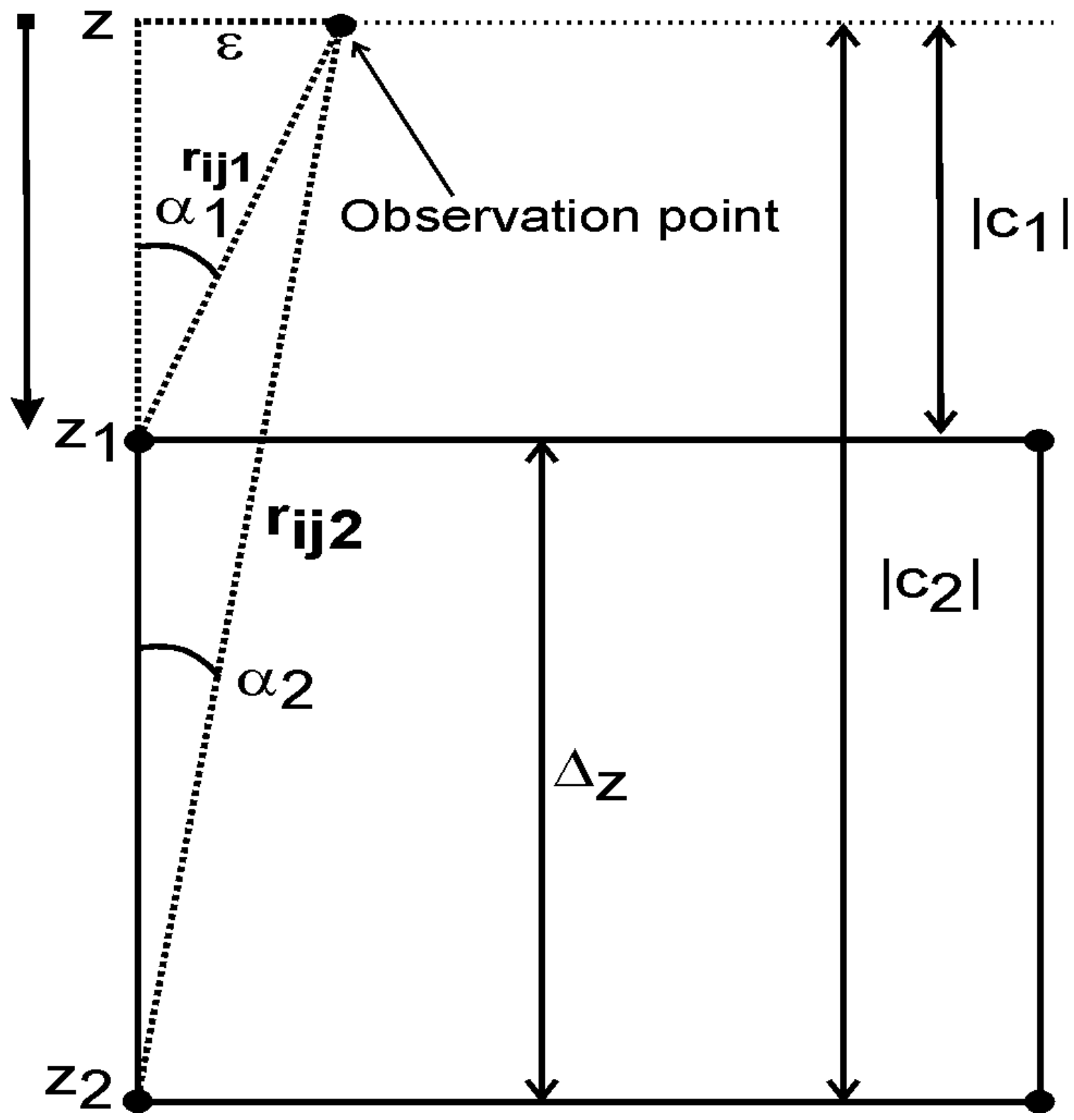

Figure 9: Computation of $g_{\ell \ell^{\prime}}$ : Illustration of the geometrical relationships used for approximating unbounded expressions in the formula for $g_{\ell \ell^{\prime}}$ by a bounded term for the case $a_{i}^{2}+b_{j}^{2}=\varepsilon \ll 1$. 


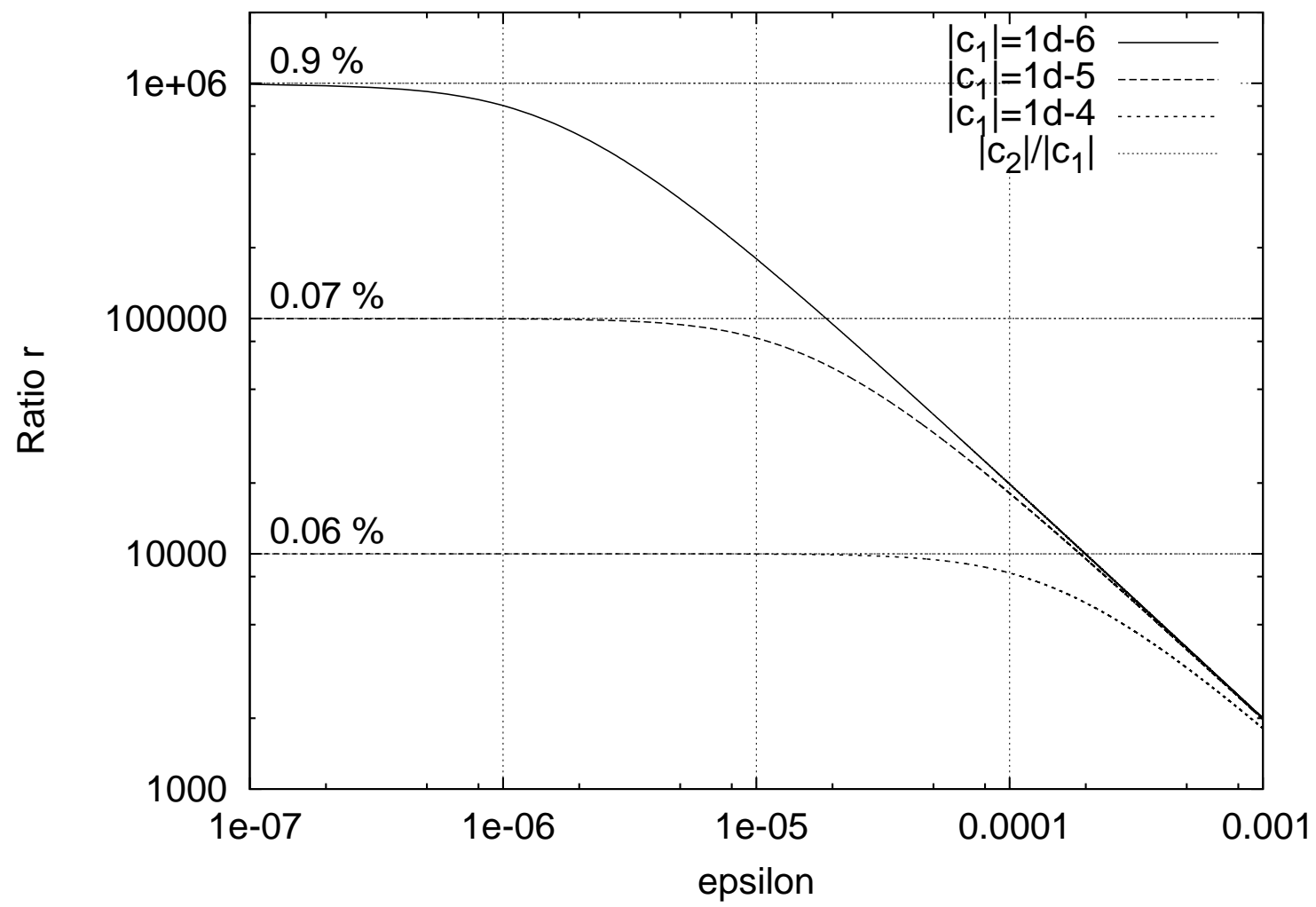

Figure 10: Computation of $g_{\ell \ell^{\prime}}$ : Plot of the ratio $r=\frac{r_{i j 1}-\left|c_{1}\right|}{r_{i j 2}-\left|c_{2}\right|}$ against $\varepsilon=a_{i}^{2}+b_{j}^{2}$ for the three different values $\left|c_{1}\right|=10^{-4} \Delta_{z}, 10^{-5} \Delta_{z}, 10^{-6} \Delta_{z}\left(\Delta_{z}=\right.$ vertical cell size). With decreasing $\varepsilon, r$ converges to the approximation (straight lines) given by (A-3). 


\section{DISCLAIMER}

This document was prepared as an account of work sponsored by the United States Government. While this document is believed to contain correct information, neither the United States Government nor any agency thereof, nor The Regents of the University of California, nor any of their employees, makes any warranty, express or implied, or assumes any legal responsibility for the accuracy, completeness, or usefulness of any information, apparatus, product, or process disclosed, or represents that its use would not infringe privately owned rights. Reference herein to any specific commercial product, process, or service by its trade name, trademark, manufacturer, or otherwise, does not necessarily constitute or imply its endorsement, recommendation, or favoring by the United States Government or any agency thereof, or The Regents of the University of California. The views and opinions of authors expressed herein do not necessarily state or reflect those of the United States Government or any agency thereof or The Regents of the University of California.

Ernest Orlando Lawrence Berkeley National Laboratory is an equal opportunity employer. 